\title{
Neurofilament light chain polypeptide gene mutations in Charcot-Marie-Tooth disease: nonsense mutation probably causes a recessive phenotype
}

\author{
Akiko Abe ${ }^{1}$, Chikahiko Numakura ${ }^{1}$, Kayoko Saito ${ }^{2}$, Hiroyoshi Koide ${ }^{3}$, Nobuyuki Oka ${ }^{4}$, Akira Honma ${ }^{5}$, \\ Yumiko Kishikawa ${ }^{1}$ and Kiyoshi Hayasaka ${ }^{1}$
}

The neurofilament light chain polypeptide (NEFL) forms the major intermediate filament in neurons and axons. NEFL mutation is a cause of axonal or demyelinating forms of dominant Charcot-Marie-Tooth disease (CMT). We investigated NEFL in 223 Japanese CMT patients who were negative for PMP22, MPZ, GJB1, LITAF, EGR2, GDAP1, MTMR2 and PRX in the demyelinating form and negative for MFN2, MPZ, GJB1, HSP27, HSP22 and GARS in the axonal form. We detected four heterozygous missense mutations-Pro8Leu, Glu90Lys, Asn98Ser and Glu396Lys-in five unrelated patients and a homozygous nonsense mutation, Glu140Stop, in one other patient. All patients had mildly to moderately delayed nerve conduction velocities, possibly caused by a loss of large diameter fibers. This is the first report of a homozygous nonsense mutation of NEFL. Results of our study show that nonsense NEFL mutations probably cause a recessive phenotype, in contrast to missense mutations, which cause a dominant phenotype.

Journal of Human Genetics (2009) 54, 94-97; doi:10.1038/jhg.2008.13; published online 16 January 2009

Keywords: Charcot-Marie-Tooth disease; NEFL; neurofilament

\section{INTRODUCTION}

Charcot-Marie-Tooth disease (CMT) is the most common inherited peripheral neuropathy affecting motor and sensory nerves of the peripheral nervous system. ${ }^{1}$ The disease has been classified into demyelinating and axonal forms based on nerve conduction velocities (NCVs). More than 26 genes have been identified as diseasecausing genes of CMT (http://www.molgen.ua.ac.be/CMTMutations/ Mutations). As a disease-causing genes of demyelinating forms, genes coding for myelin membrane proteins and proteins associated with the biology of Schwann's cells, including transcriptional proteins involved in the differentiation and development of Schwann's cells, have been identified. Regarding the axonal forms, genes coding for cytoskeletal proteins and proteins involved in axonal transport have been identified. A close relationship exists between axon and myelin forms: mutations of several genes are known to be associated with both forms.

The neurofilament light chain polypeptide (NEFL) is a constituent of neurofilaments: the major intermediate filament of neurons and axons. It plays a pivotal role in the assembly and maintenance of the axonal cytoskeleton. ${ }^{2,3}$ Neurofilaments determine the axonal diameter and the conduction velocity of peripheral nerves. ${ }^{4-6}$ Mutations of
NEFL were reported in an autosomal-dominant axonal form of CMT type $2 \mathrm{E}(\mathrm{CMT} 2 \mathrm{E})$ and $^{7}$ then in an autosomal-dominant demyelinating form of CMT type $1 \mathrm{~F}$ (CMT1F) ${ }^{8}$

We investigated the frequency and phenotypic effects of NEFL mutations in a cohort of 223 Japanese CMT patients, including those with unclassified types. We detected four heterozygous missense mutations in five patients and a homozygous nonsense mutation in one other patient. Herein, we present discussion of the genotypephenotype relation.

\section{PATIENTS AND METHODS}

\section{Patients}

We studied 223 unrelated CMT patients. On the basis of electrophysiological criteria, 121 patients were diagnosed with demyelinating CMT (motor median NCVs $<38 \mathrm{~m} \mathrm{~s}^{-1}$ ), 93 patients had axonal CMT (motor median NCVs $>38 \mathrm{~m} \mathrm{~s}^{-1}$ ) and 9 patients had unclassified CMT types. ${ }^{9}$ The latter group included patients whose detailed results of electrophysiological investigations were not available. The patients with demyelinating and unclassified CMT were all negative on mutation screening for PMP22, MPZ, GJB1, LITAF, EGR2, GDAP1, MTMR2 and PRX. The patients with axonal and unclassified types were negative for MFN2, MPZ, GJB1, HSP27, HSP22 and GARS. As controls for sequence variations, 100 healthy Japanese people were screened.

\footnotetext{
${ }^{1}$ Department of Pediatrics, Yamagata University School of Medicine, Yamagata, Japan; ${ }^{2}$ Institute of Medical Genetics, Tokyo Women's Medical University, Tokyo, Japan; ${ }^{3}$ Hallo Clinic, Saitama, Japan; ${ }^{4}$ NHO Minami-Kyoto Hospital, Kyoto, Japan and ${ }^{5}$ Honma Child Clinic, Yamagata, Japan 


\section{Genetic analyses}

The Ethics Committee of the Yamagata University School of Medicine approved this study. Peripheral blood specimens were used for genomic DNA extraction with written informed consent from the patients and their families. All exons, including the exon-intron boundaries of NEFL, were amplified using PCR with primers, as described in a previous report. ${ }^{8}$ The mutations were screened using denaturing high-performance liquid chromatography analysis (WAVE system; Transgenomic Inc., Omaha, NE, USA). The fragments showing a heteroduplex were sequenced using a cycle sequencing reaction kit and a genetic analyzer (BigDye Terminator, ABI PRISM 3100; Applied Biosystems, Foster City, CA, USA). The PCR fragments were also subcloned into a TA vector (Takara Bio Inc., Tokyo, Japan) and sequenced for confirmation.

\section{RESULTS}

\section{NEFL mutations}

Mutational analysis identified five mutations in six unrelated patients and several polymorphisms. Table 1 shows that five unrelated patients were heterozygotes of the following missense mutations: Pro8Leu (c.23C > T), ${ }^{8}$ Asn98Ser (c.293A > G), ${ }^{8,10}$ Glu90Lys (c.268G $>$ A) ${ }^{8}$ and Glu396Lys (c.1186G >A). ${ }^{11,12}$ One patient (case 2) was a homozygote of a novel nonsense mutation, Glu140Stop (c.418G $>$ T), who was born to consanguineous parents and who had a similarly affected brother. All patients except for case 2 were sporadic. The parents of case 6 , with Glu90Lys, did not carry the mutation, indicating a de novo mutation.

We also detected the following polymorphisms: c.-47_-46delTC, c. $227 \mathrm{~T}>\mathrm{A} \quad$ (Val76Ala),${ }^{10}$ c. $279 \mathrm{G}>\mathrm{A} \quad(\mathrm{Gln} 93 \mathrm{Gln}),{ }^{10}$ IVS1-24insT, IVS1-24insTT, c.1329C > A (Tyr443Tyr), c.1492G > A (Ala498Thr), ${ }^{10}$ c.1572C $>$ A (Tyr521Tyr), c.1579_1581delGAG (Glu527del) ${ }^{10}$ and c.1593T $>$ G (Val531Val).

\section{Clinical findings}

The clinical findings of the seven cases are presented in Table 1. All cases had gait disturbance before the age of 10 years and were classified as showing the demyelinating form based on a mild-to-moderate delay in motor NCVs. Four patients (cases 3, 4, 5 and 6) had very early onsets; three of them (cases 3, 4 and 6) presented with delayed motor milestones. Four patients (cases 3, 4, 5 and 6) exhibited hearing disturbance and one patient (case 4) had mental retardation. Another patient (case 5) showed pyramidal signs and cerebellar atrophy and dilatation of the fourth ventricle on brain computed tomography and magnetic resonance imaging scans.

Case 2 (a homozygote for the Glu140Stop mutation) was the second child of consanguineous parents. His parents were first cousins, but they showed no symptoms of neuromuscular disorders. His father died of prostate cancer at the age of 68 years; his mother died of gastric cancer at the age of 73 years. He had a similarly affected elder brother, who exhibited gait disturbance from his school age and used a wheelchair from the age of 40 years. He also had a healthy younger sister. The patient developed normally until the age of 2 years, when he injured his left leg. He underwent three operations for periosteomyelitis of the left femur from ages $2-4$, but precise information was not available. He showed pes cavus deformities but did not complain of significant gait disturbance. At the age of 12 years, he underwent an operation to lengthen his left Achilles' tendon because of severe toe walking, but he showed progressive muscle weakness and atrophy of the lower and upper extremities. He was unable to walk without a cane at the age of 44 years. Neurological examination at the age of 44 years showed the absence of all deep-tendon reflexes, along with muscle weakness and atrophy of the distal extremities, drop feet, pes cavus, claw hands and loss of sensation in the lower extremities. Electrophysiological examinations of median nerves showed motor NCV of $13.8 \mathrm{~m} \mathrm{~s}^{-1}$ and undetectable sensory nerve action potentials. Sural nerve biopsy revealed a loss of large- and smalldiameter fibers, in addition to small nerve clusters with a few onion bulb formations.

Table 1 Clinical feature of the examined patients

\begin{tabular}{|c|c|c|c|c|c|c|c|c|c|c|}
\hline \multirow[t]{2}{*}{$\begin{array}{l}\text { Case (mutation) } \\
\text { sex/age at present } \\
\text { (years) }\end{array}$} & \multirow{2}{*}{$\begin{array}{l}\text { Age at } \\
\text { onset } \\
\text { (years) }\end{array}$} & \multirow{2}{*}{$\begin{array}{l}\text { Initial } \\
\text { symptoms }\end{array}$} & \multicolumn{2}{|c|}{ Muscle weakness } & \multicolumn{2}{|c|}{ Atrophic features } & \multirow{2}{*}{$\begin{array}{c}\text { Sensory } \\
\text { loss }\end{array}$} & \multirow{2}{*}{ Reflexes } & \multirow{2}{*}{$\begin{array}{l}\text { Additional } \\
\text { features }\end{array}$} & \multirow{2}{*}{$\begin{array}{l}\text { MCV for } \\
\text { median } \\
\left(m s^{-1}\right)\end{array}$} \\
\hline & & & $L L$ & $U L$ & $L L$ & $U L$ & & & & \\
\hline \multicolumn{11}{|l|}{ Case 1 (Pro8Leu) } \\
\hline$F / 27$ & $<10$ & Gait problems & +2 & +1 & Yes & Yes & Yes & A & & 33.1 \\
\hline \multicolumn{11}{|c|}{ Case 2 (Glu140Stop) } \\
\hline$M / 44$ & $<10$ & Gait problems & +4 & +2 & Yes & Yes & Yes & A & Waddling gait & 13.8 \\
\hline \multicolumn{11}{|l|}{ Case 3 (Asn98Ser) } \\
\hline $\mathrm{M} / 16$ & $<2$ & Delayed walking & +1 & +1 & Yes & Yes & Yes & A & Hearing disturbance and waddling gait & 29.3 \\
\hline \multicolumn{11}{|l|}{ Case 4 (Asn98Ser) } \\
\hline $\mathrm{M} / 5$ & $<1$ & Delayed walking & ND & ND & Yes & Yes & No & A & $\begin{array}{l}\text { Mental retardation, hearing disturbance } \\
\text { and wide-based gait }\end{array}$ & 29.7 \\
\hline \multicolumn{11}{|l|}{ Case 5 (Glu396Lys) } \\
\hline$M / 34$ & $<2$ & Gait problems & +3 & +2 & Yes & Yes & Yes & $\mathrm{D}$ & $\begin{array}{l}\text { Hearing disturbance, waddling gait } \\
\text { and pyramidal sign }\end{array}$ & 35 \\
\hline
\end{tabular}

Case 6 (Glu90Lys)

$\begin{array}{lllllll}\mathrm{M} / 15 & <1 & \text { Delayed walking } & +3 & +2 & \text { Yes } & \text { Yes }\end{array}$

Abbreviations: F, female; LL, lower limbs; M, male; MRC, standard Medical Research Council scale; UL, upper limbs. Muscle weakness: ND, not determined; $+4=\mathrm{MRC} 0 ;+3=\mathrm{MRC} 1-2 ;+2=\mathrm{MR}$
Reflexes: $\mathrm{D}$, decreased; $\mathrm{A}$, absent; MCV, motor nerve conduction velocity.

${ }^{a}$ Cases $1,3,4,5$ and 6 were heterozygous for each mutation and case 2 was homozygous for a nonsense mutation. 
Sural nerve biopsy of tissues obtained from cases 3 and 5 showed a loss of large-diameter fibers with regenerated clusters. Case 6 showed a loss of large- and small-diameter fibers, small nerve clusters, endoneurial fibrosis and tomacula formation in part.

\section{DISCUSSION}

The NEFL assembles with the mid-sized neurofilament (NEFM) and heavy neurofilament (NEFH) subunits and forms the major intermediate filament in neurons. ${ }^{2,3}$ NEFL mutations were first identified in patients with the autosomal-dominant axonal form (CMT2E) and then in patients with the autosomal-dominant demyelinating form (CMT1F). ${ }^{8,7}$ More than 18 disease-causing mutations in NEFL are known; all mutations are missense mutations with the exception of the Thr21fs. ${ }^{13}$ Functional studies have shown that missense mutant NEFL proteins disrupt the assembly with wild-type NEFL and with the NEFM and NEFH, and cause aggregation, resulting in the disruption of axonal neurofilament translocation and anterograde or retrograde axonal transport including mitochondria. ${ }^{14-16}$

In this study, we found a homozygous nonsense mutation, Glu140Stop, in one patient (case 2) in addition to four heterozygous missense mutations in five other patients. The parents of case 2 were first cousins, but they never complained of muscle weakness. Case 2 had a similarly affected brother and a healthy sister. The Glu140Stop mutation might truncate the protein or might trigger nonsensemediated decay and possibly act in a loss-of-function manner. Reportedly, a quail with a homozygous nonsense mutation (Gln114Stop) of NEFL was symptomatic, but a quail with a heterozygous mutation was not. ${ }^{17}$ The pathological change of peripheral nerves in case 2 resembles that in a quail with a homozygous nonsense mutation (Gln114Stop) of NEFL and those in our patients with heterozygous missense mutations. Major pathophysiology of homozygous nonsense mutation and heterozygous missense mutation might underlie the impairment of the formation of stable neurofilament assembly. Specimens of his family members were not available; we were unable to confirm the recessive trait of this nonsense mutation through segregation studies of his family. However, the patient's information indicated that the nonsense mutation, Glu140Stop, is probably a cause of recessive CMT.

Regarding other nonsense mutations of NEFL, Glu525fs was detected in a patient from a family diagnosed with an axonal autosomal-dominant form of CMT. ${ }^{18}$ However, the Glu525fs did not co-segregate with clinical phenotype in the family; it was inferred to be a polymorphism. Actually, Glu525fs is not a disease-causing mutation in this family. It might have been detected incidentally in the patient. However, it cannot be considered simply as a polymorphism. The Glu525fs, which was not detected in 700 control chromosomes and in 84 CMT2 chromosomes, might act in a recessive manner and require another mutation in other allele to cause a disease. As another nonsense mutation, a heterozygous c.48_60dupGCGCTACGTGGAG (Thr21fs) was found in a patient with axonal CMT. ${ }^{13}$ In co-expression experiments, Thr21fs truncated NEFL prevented wild-type NEFL from forming long filaments; it also broke up the assembled NEFL filaments in a manner differing from those of other dominant missense mutations. However, the patient was an adopted child: no information related to the patient's family was available. The authors did not discount the possibility of a nonsense-mediated decay in this mutation; moreover, the possibility cannot be denied that the patient carries an unidentified NEFL mutation in other allele. Actually, Nefl knockout mice showed a morphological change in the peripheral nervous system resembling that in a quail carrying a homozygous nonsense mutation. ${ }^{19}$ However, the Nefl knockout mice developed normally and exhibited no overt symptoms. Why NEFL-deficient mice do not show symptoms similarly to the mutant quail remains an open question. One possibility is the difference in the respective sizes of the animals and another is species-related difference in neurofilament requirements during development. To clarify the phenotype of nonsense mutations in humans, data of further cases carrying nonsense mutations are necessary.

In this study, we detected NEFL mutations in about $2 \%$ of all CMT patients, as described, ${ }^{8}$ and about $3 \%$ of Japanese demyelinating CMT patients. Most of our patients with NEFL mutations presented with an early onset and moderate-to-severe symptoms. They were frequently complicated with hearing disturbance. Our six patients were diagnosed with the demyelinating form based on delayed conduction velocities. One function of neurofilaments is control of the radial growth of large myelinated axons; diameter is the principal determinant of conduction velocity in myelinated nerve fibers. ${ }^{4-6}$ Loss of large myelinated axons, as observed in our cases 2, 3, 5 and 6, might be a major cause of delayed conduction velocities. However, some patients pathologically showed thinly myelinated axons and onion bulb formation. ${ }^{8,12}$ As a possible mechanism inducing demyelination, NEFL mutations might disturb intimate axon-Schwann's cell interactions and result in secondary demyelination. Previtali et al. ${ }^{20}$ found that NEFL interacts with MTMR2 in both Schwann's cells and neurons. In addition, MTMR2 encodes a phosphatase and MTMR2 mutants cause recessive demyelinating $\mathrm{CMT}^{21}$ suggesting that NEFL mutants affect the phosphatase activity of MTMR2 and interrupt axon-Schwann's cell interactions. Another possible explanation is that NEFL is involved in the formation and maintenance of peripheral myelin in some unknown way.

Results of our study indicate that nonsense NEFL mutations probably cause a recessive phenotype, in contrast to missense mutations that cause a dominant phenotype.

\section{ACKNOWLEDGEMENTS}

This work was supported by a Grant-in-Aid for COE Research, Grant-in-Aid for Scientific Research from the Ministry of Education, Science, Culture and Sports of Japan, and grant from the Ministry of Health, Labour and Welfare of Japan.

1 Dyck, P. J., Chance, P., Lebo, R. \& Carney, J. A. Hereditary motor and sensory neuropathies. In Peripheral Neuropathy (Dyck P. J., Thomas P. K., Griffin J. W., Low P. A., Poduslo J. F. eds). 1096-1136 (Saunders, Philadelphia, 1993).

2 Lee, M. K. \& Cleveland, D. W. Neuronal intermediate filaments. Annu. Rev. Neurosci. $19,187-2171996$.

3 Grant, P. \& Pant, H. C. Neurofilament protein synthesis and phosphorylation. J. Neurocytol. 29, 843-872 2000.

4 Waxman, S. G. Determinants of conduction velocity in myelinated nerve fibers. Muscle Nerve 3, 141-150 1980.

5 Sakaguchi, T., Okada, M., Kitamura, T. \& Kawasaki, K. Reduced diameter and conduction velocity of myelinated fibers in the sciatic nerve of a neurofilament-deficient mutant quail. Neurosci. Lett. 153, 65-68 1993.

6 Eyer, J. \& Peterson, A. Neurofilament-deficient axons and perikaryal aggregates in viable transgenic mice expressing a neurofilament-beta-galactosidase fusion protein. Neuron 12, 389-405 1994.

7 Mersiyanova, I. V., Perepelov, A. V., Polyakov, A. V., Sitnikov, V. F., Dadali, E. L., Oparin, R. B. et al. A new variant of Charcot-Marie-Tooth disease type 2 is probably the result of a mutation in the neurofilament-light gene. Am. J. Hum. Genet. 67, 37-46 2000.

8 Jordanova, A., De Jonghe, P., Boerkoel, C. F., Takashima, H., De Vriendt, E., Ceuterick, C. et al. Mutations in the neurofilament light chain gene (NEFL) cause early onset severe Charcot-Marie-Tooth disease. Brain 126, 590-597 2003.

9 Harding, A. E. \& Thomas, P. K. The clinical features of hereditary motor and sensory neuropathy types I and II. Brain 103, 259-280 1980.

10 Yoshihara, T., Yamamoto, M., Hattori, N., Misu, K., Mori, K., Koike, H. et al. Identification of novel sequence variant in the neurofilament light gene in a Japanese population: analysis of Charcot-Marie-Tooth disease patients and normal individuals. J. Peripher. Nerv. Syst. 7, 221-224 2002. 
11 Choi, B. O., Lee, M. S., Shin, S. H., Hwang, J. H., Choi, K. G., Kim, W. K. et al. Mutational analysis of PMP22, MPZ, GJB1, EGR2 and NEFL in Korean Charcot-MarieTooth neuropathy patients. Hum. Mutat. 24, 185-186 2004.

12 Züchner, S., Vorgerd, M., Sindern, E. \& Schröder, J. M. The novel neurofilament light (NEFL) mutation Glu397Lys is associated with a clinically and morphologically heterogeneous type of Charcot-Marie-Tooth neuropathy. Neuromuscul. Disord. 14, 147-157 2004.

13 Leung, C. L., Nagan, N., Graham, T. H. \& Liem, R. K. A novel duplication/insertion mutation of NEFL in a patient with Charcot-Marie-Tooth disease. Am. J. Med. Genet. A 140, 1021-1025 2006.

14 Brownlees, J., Ackerley, S., Grierson, A. J., Jacobsen, N. J., Shea, K., Anderton, B. H. et al. Charcot-Marie-Tooth disease neurofilament mutations disrupt neurofilament assembly and axonal transport. Hum. Mol. Genet. 11, 2837-2844 2002.

15 Pérez-Ollé, R., López-Toledano, M. A., Goryunov, D., Cabrera-Poch, N., Stefanis, L., Brown, K. et al. Mutations in the neurofilament light gene linked to Charcot-Marie-Tooth disease cause defects in transport. J. Neurochem. 93, 861-874 2005.
16 Sasaki, T., Gotow, T., Shiozaki, M., Sakaue, F., Saito, T., Julien, J. P. et al. Aggregate formation and phosphorylation of neurofilament-L Pro22 Charcot-Marie-Tooth disease mutants. Hum. Mol. Genet. 15, 943-952 2006.

17 Ohara, O., Gahara, Y., Miyake, T., Teraoka, H. \& Kitamura, T. Neurofilament deficiency in quail caused by nonsense mutation in neurofilament-L gene. J. Cell. Biol. 121, 387-395 1993.

18 Andrigo, C., Boito, C., Prandini, P., Mostacciuolo, M. L., Siciliano, G., Angelini, C. et al. A novel out-of-frame mutation in the neurofilament light chain gene (NEFL) does not result in Charcot-Marie-Tooth disease type 2E. Neurogenetics 6, 49-50 2005.

19 Zhu, Q., Couillard-Després, S. \& Julien, J. P. Delayed maturation of regenerating myelinated axons in mice lacking neurofilaments. Exp. Neurol. 148, 299-316 1997.

20 Previtali, S. C., Zerega, B., Sherman, D. L., Brophy, P. J., Dina, G., King, R. H. et al. Myotubularin-related 2 protein phosphatase and neurofilament light chain protein, both mutated in CMT neuropathies, interact in peripheral nerve. Hum. Mol. Genet. 12, 1713-1723 2003

21 Bolino, A., Muglia, M., Conforti, F. L., LeGuern, E., Salih, M. A., Georgiou, D. M. et al. Charcot-Marie-Tooth type 4B is caused by mutations in the gene encoding myotubularin-related protein-2. Nat. Genet. 25, 17-19 2000. 